\title{
Correction to: The long-acting C5 inhibitor, ravulizumab, is efficacious and safe in pediatric patients with atypical hemolytic uremic syndrome previously treated with eculizumab
}

\author{
Kazuki Tanaka ${ }^{1} \cdot$ Brigitte Adams $^{2} \cdot$ Alvaro Madrid Aris $^{3} \cdot$ Naoya Fujita $^{1} \cdot$ Masayo Ogawa $^{4} \cdot$ Stephan Ortiz ${ }^{4}$. \\ Marc Vallee $^{4} \cdot$ Larry A. Greenbaum ${ }^{5}$
}

Published online: 9 December 2020

(C) IPNA 2020

\section{Correction to: Pediatr Nephrol.} https://doi.org/10.1007/s00467-020-04774-2

The published version of the article unfortunately contained a mistake. The baseline FACIT-Fatigue should be 50.0 (42.0$52.0)$ instead of $(42.0-50.0)$ as it was reported in the original article. The authors apologize for this mistake. The below paragraph was corrected in the original article to reflect the correct values.

The online version of the original article can be found at https://doi.org/ $10.1007 / \mathrm{s} 00467-020-04774-2$

Kazuki Tanaka

kazuki.tanaka0505@gmail.com

1 Department of Nephrology, Aichi Children's Health and Medical Center, 7-426, Morioka-cho, Obu City, Aichi prefecture 474-8710, Japan

Department of Pediatric Nephrology, Children's Hospital Queen Fabiola, Université libre de Bruxelles, Brussels, Belgium

3 Children's Nephrology and Renal Transplantation Service, Children's Maternity Hospital Sant Joan de Déu, University of Barcelona, Barcelona, Spain

4 Alexion Pharmaceuticals Inc, Boston, MA, USA

5 Division of Pediatric Nephrology, Emory University School of Medicine and Children's Healthcare of Atlanta, Atlanta, GA, USA
"The FACIT-Fatigue scores remained stable throughout the initial evaluation period and extension period up to 1 year (Supplementary Fig.3). The median (range) FACIT-Fatigue value at baseline was $50.0(42.0,52.0)$. The median change from baseline was $0.0(-5.0,3.0)$ at 26 weeks and $-1.0(-7.0$, 2.0) at 1 year."

Publisher's note Springer Nature remains neutral with regard to jurisdictional claims in published maps and institutional affiliations. 